\title{
Research on Reliability Calculation Method of Metal V-belt CVT in Sliding Friction Transmission Condition Based on the Oil Film
}

\author{
Jin-gang Liu ${ }^{1, *}$, Jian Chen $^{1}$, Dian-lun Xue ${ }^{2}$, You-hong Zhao ${ }^{1}$ \\ ${ }^{1}$ School of Mechanical Engineering, Xiangtan University, Xiangtan,411105, China \\ ${ }^{2}$ School of mechanical and vehicle engineering, Hunan University, Changsha, 410082, China
}

\begin{abstract}
Keywords: Metal V-belt CVT, Sliding Friction Transmission, Reliability, EHL, Multi-grid.
Abstract. To analyze the bearing reliability of oil film between the element and the pulley of metal V-belt cvt, a mathematical model of oil film thickness between the element and pulley was build based on the theory of EHL (elasto-hydrodynamic lubrication) and the experimental data. And then solved by multi-grid method to obtain the distribution of oil film thickness; Based on the numerical results, applying probability analysis method to study the reliability of oil film thickness. The results show that the oil film thickness distribution is approximate horseshoe, the minimum film thickness is about $0.3 \sim 0.4 \mu \mathrm{m}$; The reliability of the oil film thickness calculated by the method of probability analysis under the condition is over 0.99 , it's provided a new method to calculate the reliability of sliding friction transmission.
\end{abstract}

\section{Introduction}

Metal v-belt CVT (continuously variable transmission) owns compact structure, lower emission, good fuel economy and driving performance, leading better power matching of engine and transmission system, which is considered as the most ideal transmission scheme in hybrid car[1]. However, the efficiency of CVT transmission is lower than manual transmission, because about $30 \%$ extra clamping force was added to avoid excessive slip in transfer process caused by torque fluctuation of CVT. Excessive clamping force increases extra energy consumption of hydraulic system, and reduces the transmission efficiency[2-4]. Metal v-belt CVT transmits power through friction force, clamping force is just the external factors, the lubrication state between the element and pulley is the essence of the friction drive[5-8]. So, studying the lubrication state between element and pulley, reducing the clamping force as much as possible on the premise that the torque be transmitted correctly, improving the anti-wear ability of element and the transmitting efficiency of metal belt, which will affect the CVT 's development significantly. The reliability of sliding friction transmission has great effects on the efficiency of CVT transmission, but the clamping force control strategy in sliding friction conditions involves lots of parameters, some of which are difficult to obtain[9-12], And then, the reliability of the clamping force is hard to be assessed in a real-time. In view of this, this article established the mathematical model of film thickness of the sliding friction transmission. Based on the EHL theory, obtained the key parameters through the experiment, and used multi-grid method to solve the distribution of film thickness between the fiction blocks and the pulley, Applying the probability analysis method, the reliability of film thickness is further studied.

\section{Mathematical Model of Oil Film Thickness}

2.1 Geometric Model in Contact of the Element and the Pulley. During the calculation, according to hertz contact theory, assuming that the contact area between element and pulley are a series of ellipses, as shown in the Figure.1. And, the cross-section of contact area between the 
element and the pulley as shown in the Figure.2.

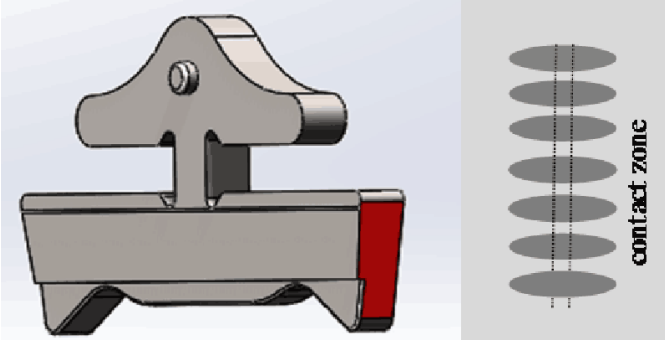

Figure.1 Geometric model in contact area

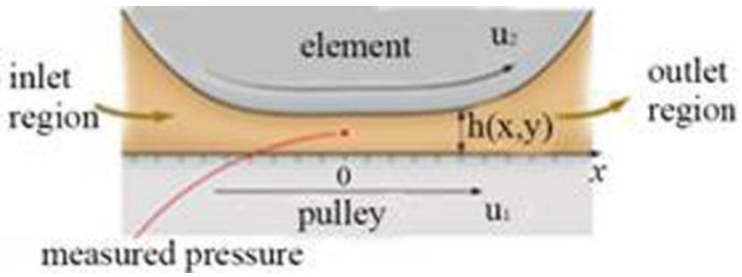

Figure. 2 Cross-section of contact area between element and pulley

2.2 Fundamental Equations. The first and the most important is Reynolds equations:

$$
\frac{\partial}{\partial x}\left(\frac{\rho h^{3}}{\eta} \frac{\partial p}{\partial x}\right)+\frac{\partial}{\partial y}\left(\frac{\rho h^{3}}{\eta} \frac{\partial p}{\partial y}\right)=12 u_{s} \frac{\partial(\rho h)}{\partial_{x}}
$$

Where $p$ is oil film pressure $(\mathrm{Pa}), h$ is oil film thickness $(\mathrm{m}), u_{s}$ is the average surface relative $\operatorname{speed}(\mathrm{m} / \mathrm{s}), \eta$ is the dynamic viscosity of lubricating oil $(\mathrm{Pa} \cdot \mathrm{s}), \rho$ is the density of lubricating oil $\left(\mathrm{kg} / \mathrm{m}^{3}\right)$.

In order to apply Reynolds equations correctly in this paper, some assumptions are put forward as follows:

a). Ignore the volume force and inertial force. b). The velocity of oil which contacted to the surface is the same as the velocity of contact surface. c). The lubrication film pressure along the thickness direction does not change. d). Lubricating oil is incompressible Newtonian fluid, and it is laminar flow.

The boundary conditions of Reynolds equations are as follows:

$$
\left\{\begin{array}{c}
p=0, x=x_{\text {in }} \\
p=\frac{d p}{d x}=0, x=x_{\text {out }}
\end{array}\right.
$$

Oil film thickness equation is:

$$
h=h_{0}+\frac{x^{2}}{2 R_{x}}+\frac{y^{2}}{2 R_{y}}+\frac{2}{\pi E} \iint_{\Omega} \frac{p(s, t) d s d t}{\sqrt{(x-s)^{2}+(y-t)^{2}}}
$$

Where $h$ is the nominal film thickness; $h_{0}$ is the central oil film thickness; $R_{x}, R_{y}$ is respectively the radius of curvature between the two contact surfaces; $E$ is the comprehensive elastic modulus of materials, which can be obtained by the following equation:

$$
\frac{2}{E}=\frac{1-v_{1}^{2}}{E_{1}}+\frac{1-v_{2}^{2}}{E_{2}}
$$

Where, $v_{1}, v_{2}$ is respectively Poisson ratio of the two contact bodies.

2.3 Lubricating Oil Viscosity-Pressure and Density-Pressure Equation. The relation of the lubricating oil viscosity and pressure is:

$$
\eta=\eta_{0} \exp \left\{\left(\ln \eta_{0}+9.67\right) \times\left[\left(1+5.1 \times 10^{-9} p\right)^{0.68}-1\right]\right\}
$$

Where, $\eta$ is the viscosity at the pressure $p, \eta_{0}$ is the viscosity at standard atmospheric pressure. Lubricating oil density - pressure equation 


$$
\rho(p)=\rho_{0} \frac{5.9 \times 10^{8}+1.34 p}{5.9 \times 10^{8}+p}
$$

Where, $\rho_{0}$ is the density at the environment pressure.

\section{Related Parameters}

3.1 The Oil Film Pressure Between the Belt and Pulley. The most important parameters for model is the value of pressure $p$. With the lesson of sliding bearing oil film thickness measurement method [13], a film sensor was implanted on the pulley, and override PVD (Physical Vapor Deposition), obtain the pressure data between the element and the pulley. The whole experiment device of a two-dimensional diagram is as Figure.3.

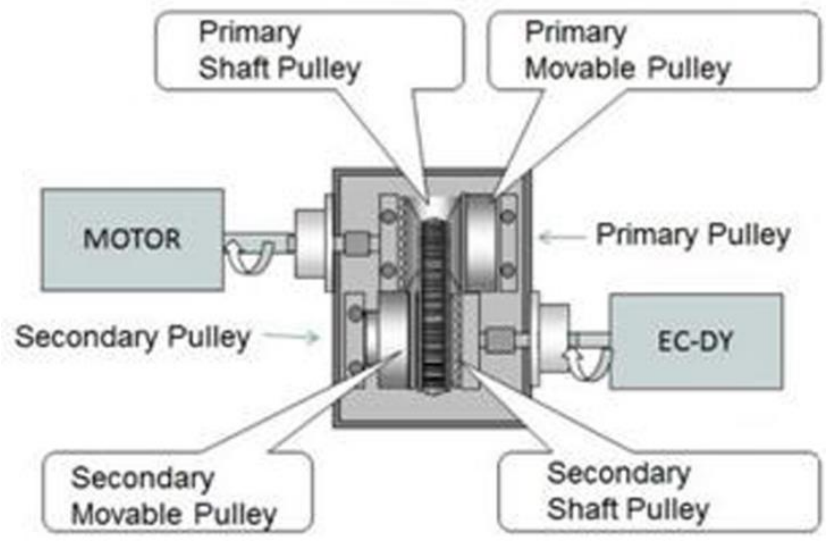

Figure. 3 the whole experiment set
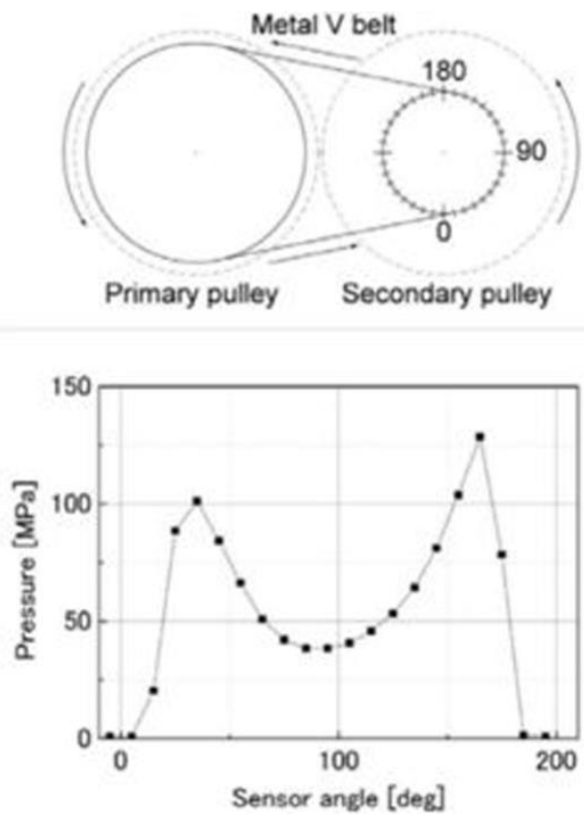

Figure.4 Measurements of the oil film pressure

3.2 Experimental Conditions. The parameters of the metal belt of the CVT used in the experiment is as shown in the following Table 1. In the experiments, the sensor is installed on the secondary pulley, the rotate speed of the primary pulley is fixed $(3000 \mathrm{rpm})$, the torque of the primary pulley is also fixed $(100 \mathrm{~N} \cdot \mathrm{m})$, the experimental measurement is the oil film pressure value between the metal belt and the secondary pulley.

Table 1. parameters of the CVT

\begin{tabular}{|c|c|}
\hline parameters & Value \\
\hline Speed ratio range & $0.442 \sim 2.432$ \\
\hline Pulley half cone angle $\left[^{\circ}\right]$ & 11 \\
\hline Pulley center distance $\mathrm{A}[\mathrm{mm}]$ & 146 \\
\hline Metal belt length $\mathrm{L}[\mathrm{mm}]$ & 615.4 \\
\hline
\end{tabular}

The parameters of the elements and the pulley material are shown in the Table 2 .

Table 2. Material parameters of the element and pulley

\begin{tabular}{|c|c|c|}
\hline Parameters & element & pulley \\
\hline Material & Rolling bearing steel & 18Ni maraging steel \\
\hline Modulus of elasticity [GPa] & 208 & 192 \\
\hline Poisson's ratio & 0.31 & 0.30 \\
\hline
\end{tabular}


The lubricating oil parameters used are shown in the Table 3.

Table 3. Parameters of the lubricating oil

\begin{tabular}{|c|c|c|c|}
\hline Parameters & Temperature $\left[{ }^{\circ} \mathrm{C}\right]$ & test method & value \\
\hline Density $\left[\mathrm{kg} / \mathrm{m}^{3}\right]$ & 15 & DIN51755 & 852 \\
\hline \multirow{3}{*}{ Viscosity $\left[\mathrm{mm}^{2} / \mathrm{s}\right]$} & 100 & ASTM D445 & 7.0 \\
\cline { 2 - 4 } & 40 & ASTM D445 & 30.3 \\
\cline { 2 - 4 } & -40 & ASTM D2983 & 9800 \\
\hline
\end{tabular}

The results are shown in the Figure. 4

\section{Model Solution and Analysis}

4.1 The Mathematical Model of Dimensionless. The multi-grid method is adopted to solve the model, first, dimensionless equations are obtained.

The dimensionless Reynolds equation is:

$$
\frac{\partial}{\partial X}\left[\varepsilon \frac{\partial P}{\partial X}\right]+\frac{\partial}{\partial Y}\left[\varepsilon \frac{\partial P}{\partial Y}\right]-\frac{\partial\left(\rho^{*} H\right)}{\partial X}=0
$$

The boundary conditions are as follows:

The inlet and outlet region:

$$
\left\{\begin{array}{c}
P\left(X_{\text {in }}, Y\right)=0 \\
P\left(X_{\text {out }}, Y\right)=0, \frac{\partial P\left(X_{\text {out }}, Y\right)}{\partial X}=0
\end{array}\right.
$$

The film thickness equation is:

$$
H(X, Y)=H_{0}+\frac{X^{2}+Y^{2}}{2}+\frac{2}{\pi} \int_{x_{\text {in }}}^{x_{\text {out }}} \int_{y_{\text {in }}}^{y_{\text {out }}} \frac{P(S, T) d S d T}{\sqrt{(X-S)^{2}+(Y-T)^{2}}}
$$

The density-pressure and viscosity-pressure equation are:

$$
\rho^{*}=\frac{5.9 \times 10^{8}+1.34 P p_{0}}{5.9 \times 10^{8}+P p_{0}} ; \quad \int_{x_{\text {in }}}^{x_{\text {out }}} \int_{y_{\text {in }}}^{y_{\text {out }}} P(X, Y) d X d Y=\frac{2}{3} \pi
$$

4.2 The Results and Analysis. Using multi-grid method with computer programming to solve the above equations, the results are as follows. Oil film thickness distribution is shown in Figure.5.

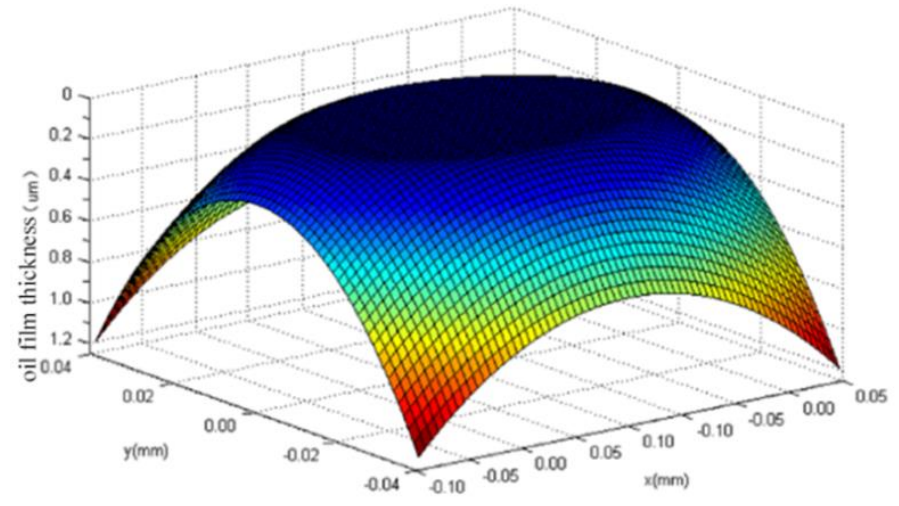

Figure.5 map of oil film thickness

Take $y=0 \mathrm{~mm}$, the distribution of oil film thickness along the direction $x$ is as shown in the Figure.6: 


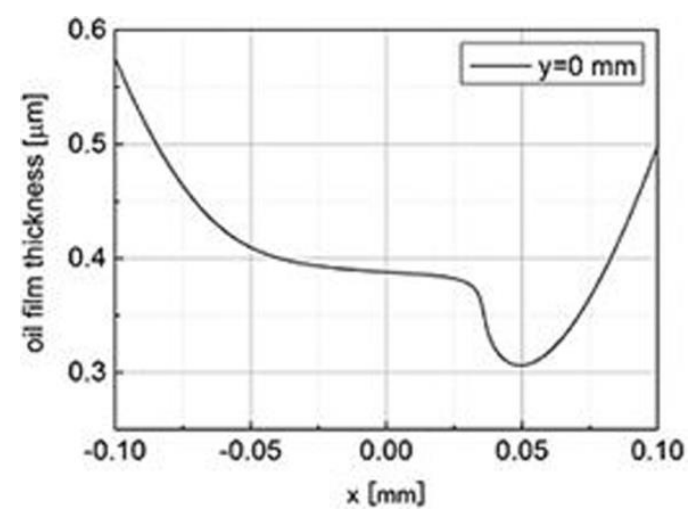

Figure.6 oil film thickness distribution when

$$
y=0 \mathrm{~mm}
$$

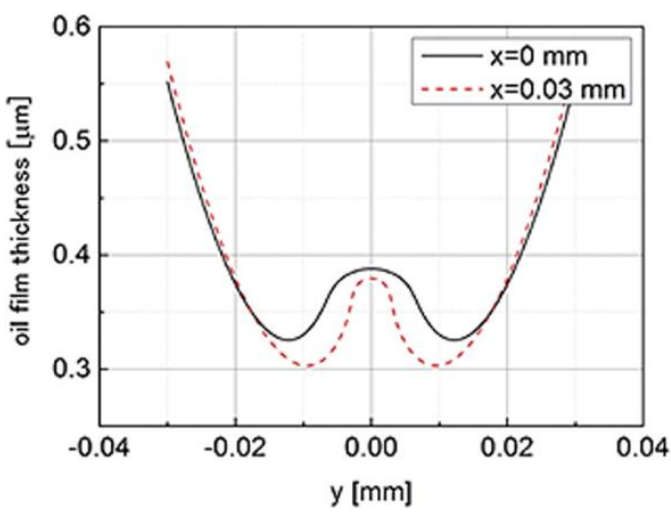

Figure.7 Oil film thickness distribution when $x=0 \mathrm{~mm}, x=0.03 \mathrm{~mm}$

Take $x=0 \mathrm{~mm}$ and $x=0.03 \mathrm{~mm}$, the distribution of oil film thickness along the direction $y$ is as shown in Figure.7.

In Figure.5, the positive direction of the axis $x$ represents the rotation direction of the pulley, which is orthogonal to the horizontal axis $y$. The figure shows that the spatial distribution of the film thickness is approximate horseshoe. The oil film thickness is about $0.5 \mu \mathrm{m}$ at $x=-0.01 \mathrm{~mm}$, which corresponds to the condition that the pulley is in contact with the element. The point $x=0 \mathrm{~mm}, y=$ $0 \mathrm{~mm}$ corresponds to the condition that the contact pressure is up to be local maximum value (Figure. 4 first pressure spike), at this point the oil film thickness is about $0.4 \mu \mathrm{m}$. In the vicinity of the oil film thickness, the thickness of the oil film decreases along the direction $x$, and the minimum oil thickness appears in the vicinity of $x=0.03 \mathrm{~mm}$.

The film thickness distribution shown in Figure.5 is based on the contact pressure of experiment measurements between the metal belt and the pulley, indicating that the lubrication between the belt and the pulley can be more accurate quantitative analysis. Figure.6 shows that the minimum film thickness is about $0.3 \mu \mathrm{m}$, which indicates that the film thickness is in the EHL film thickness range $(0.1 \mu \mathrm{m} \sim 1 \mu \mathrm{m})$ when the secondary pulley and the metal belt is in friction transmission condition.

\section{Reliability Analysis of Film Thickness}

By numerically solving the EHL equations, the distribution of oil film thickness between the element and the pulley is obtained. The calculation results are accurate, however, they only show the characteristics of average value of oil film thickness, which cannot reflect the influence of the fluctuations of calculation parameters such as the load, the surface relative movement speed and other factors. When considering the micro morphology of the contact surface, in order to guarantee the lubrication between the element and pulley to be in EHL, and for further designing CVT control strategy, it is necessary to further study the reliability of the oil film thickness.

5.1 Mathematical Model of Reliability of Oil. For the random variable $X$ with the mean value $\mu_{x}$ and standard deviation $\sigma_{x}$, the variation coefficient can be defined as:

$$
C_{x}=\frac{\sigma_{x}}{\mu_{x}}
$$

For general multivariable functions

$$
z=a_{0} \cdot x_{1}^{a_{1}} \cdot x_{2}^{a_{2}} \cdots x_{n}^{a_{n}}
$$

The variation coefficients is 


$$
C_{z}^{2}=a_{1}^{2} C_{X_{1}}^{2}+a_{2}^{2} C_{X_{2}}^{2}+\cdots a_{n}^{2} C_{X_{n}}^{2}=\sum_{i=1}^{n} a_{i}^{2} C_{X_{i}}^{2}
$$

The study conducted by Dowson, D. and Higginson, G.R[14,15] shows that:

$$
h_{\min }=2.65 \frac{\alpha^{0.54}\left(\eta_{0} u\right)^{0.7} R^{0.43}}{E^{0.03} w^{0.13}}
$$

where, $\alpha$ is the viscosity-pressure coefficient, and $w$ is the load parameter, and the other parameters are the same as the previous ones.

By the above formula,

$$
C_{\bar{h}} \approx\left(0.54^{2} C_{\alpha}^{2}+0.7^{2} C_{u}^{2}+0.43^{2} C_{R}^{2}+0.03^{2} C_{E}^{2}+0.13^{2} C_{w}^{2}\right)^{0.5}
$$

In engineering conditions the change range of the random variables $\alpha, R$, Eare very small, thus can be neglected, then equation (15) can be written as

$$
C_{\bar{h}} \approx\left(0.7^{2} \cdot \mathrm{C}_{u}^{2}+0.13^{2} \cdot \mathrm{C}_{w}^{2}\right)^{1 / 2}
$$

The standard deviation of the minimum oil film thickness is:

$$
\sigma_{\bar{h}}^{2}=C_{\bar{h}}^{2} \bar{h}^{2}
$$

In the operation process of metal V-belt and pulley, the greater the value of the minimum oil film thickness is, the higher the reliability of the oil film is. In order to objective and quantitative calculate the surplus capacity of the minimum oil film thickness, according to the interference probability principle, the reliability of the minimum oil film thickness connection equation can be established as follows:

$$
Z_{R}=\frac{\bar{h}_{s}-\bar{h}}{\left(\sigma_{\bar{h}_{s}}^{2}+\sigma_{\bar{h}}^{2}\right)^{1 / 2}}
$$

It can also be written as

$$
\frac{\bar{h}_{s}}{\bar{h}}=\frac{1+Z_{R}\left(C_{\bar{h}_{s}}^{2}+\mathrm{C}_{\bar{h}}^{2}-\mathrm{Z}_{\mathrm{R}}^{2} \mathrm{C}_{\bar{h}}^{2} C_{\bar{h}_{s}}^{2}\right)^{1 / 2}}{1-\mathrm{Z}_{\mathrm{R}}^{2} C_{\bar{h}_{s}}^{2}}
$$

\subsection{Calculation and Analysis of Reliability of Oil Film Thickness}

5.2.1 Mean Value, Standard Deviation and Coefficient of Load $w$. The peak pressure between the belt and the pulley obtained from the experiments is $120 \mathrm{MPa} 150 \mathrm{MPa}$, combining with the measured element size, concluding that,

$$
\sigma_{w}=\frac{w_{\max }-w_{\min }}{6}=200 N ; \quad C_{w}=\frac{\sigma_{w}}{\bar{w}}=0.0370
$$

5.2.2 Mean Value, Standard Deviation and Coefficient of Relative Velocity $u$. According to the relative velocity variation range $8.85 \sim 13.2 \mathrm{~m} / \mathrm{s}$, concluded that,

$$
\sigma_{u}=\frac{u_{\max }-u_{\min }}{6}=0.725 \mathrm{~m} / \mathrm{s} ; \quad C_{u}=\frac{\sigma_{u}}{\bar{u}}=0.0329
$$

\subsubsection{Mean Value, Standard Deviation and Coefficient of Minimum Oil Film Thickness}

$$
\bar{h}=2.65 \frac{\alpha^{0.54}\left(\eta_{0} u\right)^{0.7} \mathrm{R}^{0.43}}{E^{0.03} w^{0.13}}=0.42 \mu m
$$




$$
\begin{aligned}
& C_{\bar{h}} \approx\left(0.7^{2} C_{u}^{2}+0.13^{2} C_{w}^{2}\right)^{0.5}=0.0235 \\
& \sigma_{h}=C_{h} \bar{h}=0.00987 \mu \mathrm{m}
\end{aligned}
$$

5.2.4 Mean Value, Standard Deviation and Coefficient of The Oil Film Thickness Calculated From the Surface Roughness. Surface roughness of pulley is $\mathrm{R}_{1}=0.2 \sim 0.6$, Surface roughness of element is $\mathrm{R}_{2}=0.4 \sim 1.2$. The standard deviation of the roughness is

$$
\begin{array}{ll}
\sigma_{R 1}=\left(R_{1 \max }-R_{1 \min }\right) / 6=0.0667 ; & \sigma_{R 2}=\left(R_{2 \max }-R_{2 \min }\right) / 6=0.1333 \\
\sigma_{s}^{2}=4\left(\sigma_{R 1}^{2}+\sigma_{R 2}^{2}\right)=0.0889 ; & C_{s}^{2}=\sigma_{s}^{2} / \bar{h}_{s}^{2} \\
\bar{h}_{s}=\frac{1}{2}\left(R_{1 \max }+R_{2 \min }\right)=0.3 \mu \mathrm{m} &
\end{array}
$$

Therefore, $\quad C_{s}^{2}=\sigma_{s}^{2} / \bar{h}_{s}^{2}=0.9875$

\subsubsection{Take the Reliability of Minimum Oil Film Thickness as 0.99, then}

$$
\bar{h}_{99}=\frac{\bar{h}+\bar{h} Z_{99}\left(C_{s}^{2}+C_{h}^{2}-Z_{99}^{2} C_{s}^{2} C_{h}^{2}\right)}{1-Z_{99}^{2} C_{s}^{2}}=0.36 \mu m
$$

The results show that the average value of the theoretical minimum oil film thickness is $\bar{h}=0.42 \mu \mathrm{m}$, and the limiting minimum oil film thickness is $\bar{h}_{s}=0.3 \mu \mathrm{m}$, thus $\bar{h}>\overline{h_{s}}$ and $\bar{h}>\overline{h_{99}}$. Namely, under this condition, the reliability of minimum oil film thickness is above 0.99 , so the oil-film thickness between pulley and element has high surplus capacity.

\section{Conclusions}

(1) Measured the contact pressure between element and pulley by experiment, built a model based on EHL theory. the lubricating oil film between the belt and the pulley can be quantitatively analyzed by numerical calculation, the minimum oil film thickness is about $0.3 \mu \mathrm{m}$ in this paper, which is in the range of film thickness of EHL oil film thickness $(0.1 \mu \mathrm{m} \sim 1 \mu \mathrm{m})$.

(2) Considering the surface topography and other conditions of the metal V-belt and the pulley, the reliability of oil film thickness can be calculated by the method of variation coefficient. The EHL oil film between the metal belt and the pulley has high surplus capacity in this paper.

(3) Through the analysis of the minimum oil film thickness formula proposed by Dowson, D. and Higginson, G.R. combined with probability analysis method, the control strategy of the clamping force can be further studied based on the EHL oil film thickness reliability, thus to improve the transmission efficiency of CVT system.

\section{Acknowledgement}

This project was financially supported by National Natural Science Foundation of China (Grant No.51475402 and No.51575166) and key project of Science Research of Hunan Provincial Department of Education (Grant No.15A185).

\section{References}

[1] Feng, K. 2009. The application of CVT transmission system integrated with ISG in HEV[D]. Hunan University.

[2] Zhang, Y. 2013.Research on the slip ratio control for V-belt CVT[D]. Hunan University. 
[3] Cao, C.L. Zhou, Y.S. Gao S. An, Y. 2010. A study on clamping force test of metal V-belt type CVT. Journal of Hunan University 37(7): 23-26.

[4] Cao, C.L. Zhou, Y.S. Gao, S. An, Y. 2012. Study on clamping force of metal V-belt type CVT based on slip control. China Mechanical Engineering 23(23): 2893-2897.

[5] Guebeli, M.Micklem, J.D. Burrows, C.R. 1993. Maximum transmission efficiency of a steel belt continuously variable transmission. Journal of Mechanical Design 115(4): 1044-1048.

[6] Micklem, J.D. Longmore, D.K. Burrows, C.R. 1994. Modelling of the steel pushing v-belt continuously variable transmission. Proceedings of the Institution of Mechanical Engineers, Part C: Journal of Mechanical Engineering Science 208(1): 13-27.

[7] Carbone, G.Mangialardi, L.Mantriota, G. 2005. The influence of pulley deformations on the shifting mechanism of metal belt CVT. Journal of mechanical design 127(1): 103-113.

[8] Carbone, G. De, N.L. Commissaris,G.2010. An enhanced CMM model for the accurate prediction of steady-state performance of CVT chain drives. Journal of Mechanical Design 132(2): 021005.

[9] Gerbert, B.G. 1972. Force and slip behavior in V-belt drives. ActaPolytechnicaScandinavica-Mechanical Engineering Series (67): 1-10.

[10]Gerbert, B.G. 1984. Metal V-belt mechanics. Design Automation Conference, Advances in Design Automation, ASME, Boston, MA.

[11]Ashloy, E. 1994. CVT: The Car Transmission of Future. Mechanical Engineering 116 (11): 65-68.

[12]Pennings, B.Drogen, M.Brandsma, A. Van, D.M.2003. CVT Fluid Test-A Test Method on Belt-Pulley Level to Select Fluids for Push Belt CVT Application. SAE Paper : 01-3253.

[13]Mihara, Y. Hayashi, T. Nakamura, M. 1995. Development of measuring method for oil film pressure of engine main bearing by thin film sensor. JSAE review 16(2): 125-130.

[14]Dowson, D. Higginson, G.R. 1959. A numerical solution to the elasto-hydrodynamic problem. Journal of mechanical engineering science 1(1): 6-15.

[15]Dowson, D. Higginson, G.R.2014.Elasto-hydrodynamic lubrication: international series on materials science and technology. Elsevier. 